\title{
Whole virus detection using aptamers and paper-based sensor potentiometry
}

\author{
Subhashish Dolai and Massood Tabib-Azar * \\ University of Utah \\ * Correspondence: azar.m@utah.edu
}

\begin{abstract}
Paper-based sensors, microfluidic platforms and electronic devices have attracted attention in the past couple of decades because they are flexible, can be recycled easily, environmentally friendly, and inexpensive. Here we report a paper-based potentiometric sensor to detect the whole Zika virus for the first time with a minimum sensitivity of $2.6 \mathrm{nV} / \mathrm{Zika}$ and a minimum detectable signal (MDS) of $1.2 \times 10^{6} \mathrm{Zika}$. Our paper sensor works very similar to a P-N junction where a junction is formed between two different wet regions with different electrochemical potentials near each other on the paper. These two regions with slightly different ionic contents, ionic species and concentrations, produce a potential difference given by the Nernst equation. Our paper sensor consisted of a 2-3 $\mathrm{mm} \times 10 \mathrm{~mm}$ segments of a paper with a conducting silver paint contact patches on two ends. The paper is dipped in a buffer solution containing aptamers designed to bind to the capsid proteins on Zika. Atomic force microscopy and Raman spectroscopy were carried out to verify both the aptamer and Zika become immobilized in the paper. We then added the Zika (in its own buffer) to the region close to one of the silver-paint contacts. The Zika virus ( $40 \mathrm{~nm}$ diameter with $43 \mathrm{kDa}$ or $7.1 \times 10^{-20} \mathrm{gm}$ weight) became immobilized in the paper's pores (as shown using AFM and Raman spectra) and bonded with the resident aptamers creating a concentration gradient. The potential measured between the two silver paint contacts reproducibly became more negative upon adding the Zika. We also showed that an LCD powered by the sensor, can be used to detect the sensor output.
\end{abstract}

Keywords: Paper based sensor; whole virus; Zika; Aptamer.

\section{Introduction}

Sensors built with paper as substrate have short response time, are low-cost [1] and they are flexible [2], [3]. Moreover, they are biodegradable and suitable for mass deployment in resource limited areas and can be easily used by unskilled operators. Paper is also great medium for immobilizing and trapping and in some cases binding with biomolecules. Its porous structure with large connected pores composed of cellulose fibers allows to transport liquid by means of capillary forces that result in short response time. The porous structure also allows any particle or species to remain immobilized in the paper structure. Paper can also be functionalized with certain materials for selective sensing like nitrocellulose paper used for immobilizing nucleic acids [4]. Paper-based potentiometric sensors have been reported for detecting many ions and proteins [4]-[7]. Potentiometric paper-based sensors [1], [8]-[16] utilize gradients of ion distributions that generate open circuit voltages $\left(\mathrm{V}_{\text {oc }}\right)$ to transduce analytes.

There are many Zika sensors and detection methods reported in the literature. These include serum analysis using the antibody detection assays [17], [18] and detection of viral RNA using molecular-based techniques like conventional polymerase chain reaction (PCR). Real-time reverse transcription polymerase chain reaction (RT-PCR) [19]-[25] to sense Zika has also been reported. These techniques involve using viral antibody or DNA/RNA extraction followed by labelled detection using fluorescent probes. They provide high sensitivity and specificity but require specialized equipment and expensive procedures and are time consuming. The paper-based sensors are fast, inexpensive and can be used in regions with limited resources. We show that a simple LCD 
can be used to measure the sensor output with the power generated with the sensor itself. The paper sensor reported here can be modified by replacing its aptamer with aptamers for other viruses, such as corona virus, pathogens and even bacteria.

Our work shows for the first time that a potentiometric paper sensor can reliably detect a whole virus using standard printer papers. Paper can be functionalized with antigens or aptamers. Aptamers are single strand DNA structures that are artificially grown to detect biomolecules and pathogens with high specificity [26], [27]. In our sensors, the paper is functionalized with Zika aptamer and when Zika was added to one side of the paper, it resulted in a concentration gradient with the associated electrochemical potential difference. Electrical contacts to the paper sensor can be made with Graphene [15], [16] conducting glues and epoxies or silver paint that was used here. We also developed a proof-of-concept device (Fig. 2(a)) that can be printed with silver paint contacts or any other conducting inks. We also showed that the paper-based Zika sensor can be connected to an LCD for electronic readout.

\section{Theoretical Background}

The charge distribution and ionic transport of different charged species in the paper device and the resulting open circuit voltage, can be explained using phase boundary model formed by two different ionic species (aptamer in the background and Zika added on the positive side). The electrochemical potential difference is a sum of the phase boundary potentials [28] between the Zika/aptamer-electrode $\left(\mathrm{V}_{\mathrm{oc}}\right)$, the liquid junction potential [29], [30] between the Zika/aptameraptamer $\left(\mathrm{V}_{\mathrm{oc}}\right)$ and between the aptamer-electrode due to concentration difference of the Zika between the reference aptamer-buffer soaked paper $\left(\mathrm{V}_{\text {ocs }}\right)$, results in the sum-total open circuit voltage or the zero-current potential $\left(\mathrm{V}_{\mathrm{oc}}=\mathrm{V}_{\mathrm{oc} 1}+\mathrm{V}_{\mathrm{oc} 2}+\mathrm{V}_{\mathrm{oc}}\right)$. Fig. 1 shows the schematic of the phase boundary between electrodes and the paper and liquid junction potential at the center. The phase boundary potential between the Zika/aptamer-electrode interface is obtained from the Nernst equation and has a logarithmic dependence with the concentration of Zika sample.

$$
\begin{gathered}
{\left[\mathrm{Zika}^{-}\right] \rightleftharpoons[\mathrm{Zika}]+e^{-}} \\
V_{o c 1}=V_{0}-\frac{R T}{n_{1} F} \ln \frac{1}{\left[Z i k a^{-}\right]} .
\end{gathered}
$$

where, $V_{0}$ is the constant standard potential $(\mathrm{V}), \mathrm{R}$ is the gas constant, $\mathrm{T}$ is temperature $(\mathrm{K}), \mathrm{F}$ is the faraday constant $(\mathrm{C} / \mathrm{mol})$ and $\mathrm{n}_{1}$ is the number of electron $(\mathrm{mol})$. The phase boundary potential at the aptamer-electrode interface is similar to above expression and is given by, $V_{o c 3}=V_{0}-$ $\frac{R T}{n_{3} F} \ln \frac{1}{[\text { aptamer }]^{-}}$, where the symbols have their usual meaning. The liquid junction potential between Zika/aptamer-aptamer can be written in the form [31, 32],

$$
V_{o c 2}=-\sum_{i} \frac{R T}{F z_{i}} \int_{1}^{m} t_{i} d\left(\ln C_{i}\right)
$$

where, $z_{i}$ is the signed valence of the particular sample species, $C_{i}$ denotes the activity of the ions/sample species and $t_{i}$ is the transference number and signifies the fractional conductance of the $i^{\text {th }}$ ion/sample species. The transference number defined by $t_{i}=\frac{\left|z_{i}\right| u_{i} M_{i}}{\sum_{j}\left|z_{j}\right| u_{j} M_{j}}$, where $u$ is the mobility of the $i^{\text {th }}$ ion and $M$ is the molar concentration, with $j$ ranging over all the ions. An electro neutrality region is assumed to be formed, as the Zika is added on one side. The neutral region is formed by charge separation of the negatively charged Zika and the cations across the junction interface due to their different free energies in the two phases [28]. As can be seen in Fig. 4, the buffer has positive residual charge. On the other hand, the Zika has negative residual charge (Fig. 5). A net electric dipole forms between the positive buffer and nearly zero charge density buffer + Zika regions where the compensating positive charges of the buffer cancels the negative charge of the Zika or Zika/aptamer conjugate. This resulted in different $\Delta \mathrm{V}_{\mathrm{oc}}$ for Zika only and Zika/aptamer conjugate. The Zika/aptamer conjugate provided higher $\mathrm{V}_{\text {oc }}$ change than Zika only. The reason can be attributed to the aptamer configuration change and charge redistribution after binding with Zika virus. 
Additionally, Zika (40 $\mathrm{nm}$ diameter) becomes immobilized in the paper fibers with the aptamers (reason discussed earlier with the AFM studies), hence there is no diffusion of Zika species through the interface, which allows phase boundary model to be implemented in this scenario. The selectivity of Zika is addressed with the use of Zika aptamer in section 4 .

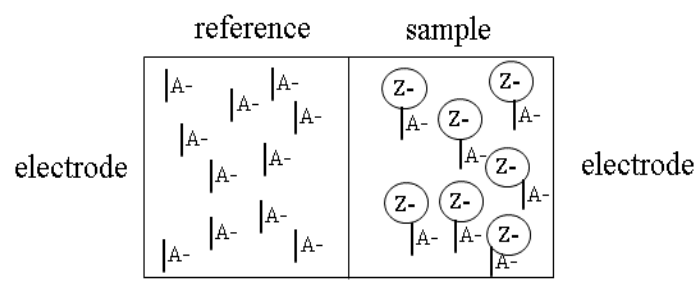

Figure 1. Schematic of the phase-boundary potential model with the electrodes and liquid wherein the potential is given by uneven distribution of the sample ions/particles.

\section{Materials and Methods:}

The devices used in our work (Fig. 2) consisted of a sample holder with two clips and electrodes mounted on a glass slide. The devices were then connected to a data acquisition system (NI-USB 6341 DAQ) and a computer with custom LabVIEW program (Fig. 2b). We measured the open circuit voltage $\left(V_{o c}\right)$ of the paper device connected with the two clips as a function of time. The glass slide was located inside a grounded copper enclosure to shield and improve the signal-to-noise ratio. Paper strips $(0.2-0.3 \mathrm{~cm} \times 1 \mathrm{~cm})$ used in our devices were manually cut from a standard printer paper and were contacted with silver paint (from Ted-Pella). These paper devices were then coated with appropriate analytes such as de-ionized (DI) water/buffer/aptamer and buffer/Zika solutions.

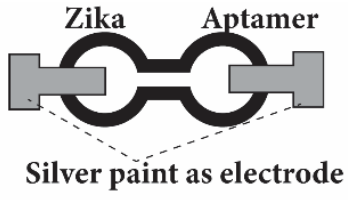

(a)

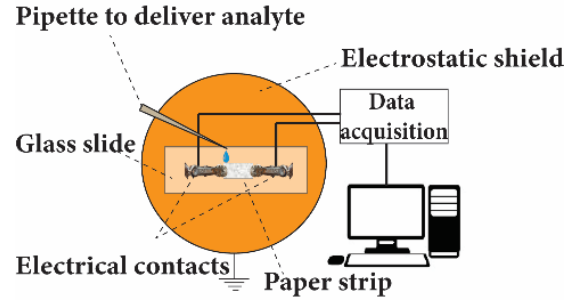

(b)

Figure 2. Schematic of the (a) proposed printed paper device. (b) experimental setup.

We used a 1x concentration of Phosphate Buffer Solution (PBS) with $1 \mathrm{mM}$ Magnesium Chloride as a buffer solution. The Zika aptamers were reconstituted as per BasePair Biotechnologies Inc. by mixing the dried aptamers in resuspension buffer also provided by the manufacturer. The aptamer solution was then diluted to working concentration of $100 \mu \mathrm{M}$ using an aptamer folding buffer followed by heating to $90^{\circ} \mathrm{C}-95^{\circ} \mathrm{C}$ for 5 minutes. The resulting aptamer solution was then diluted to $1 \mu \mathrm{M}$ solution using the buffer solution. $1 \mu \mathrm{M}$ aptamer had $\sim 1.2 \times 10^{12}$ aptamers in $2 \mu$ volume (calculated from the Avogadro number present in $1 \mathrm{M}$ concentration in 1 liter). The aptamer used in our experiments had a thiol end group and consisted of 32 nucleotide-chain that folded to bind with Zika SF9 envelope protein [31]. Fig. 3 shows the schematic of the predicted structure of the aptamer. The Zika was obtained from Zeptometrix and was diluted with $40 \mu \mathrm{l}$ of TCID_50 Zika stock solution in $60 \mu \mathrm{l}$ buffer. The number of Zika viruses in $2 \mu \mathrm{l}$ volume was $\sim 7 \times 10^{6}$, estimated in same manner as in [32]. 


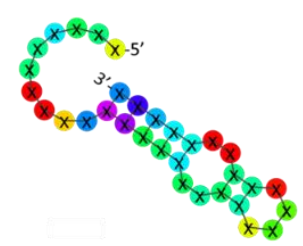

Figure 3. Predicted structure of the aptamer (Image courtesy: BasePair Biotechnologies Inc. [33])

\section{Results and Discussions}

The open circuit voltage $\left(\mathrm{V}_{\text {oc }}\right)$ of a water-coated paper device with the buffer solution introduced on the positive contact side is shown in Fig. 4 as a function of buffer concentration. The $\mathrm{V}_{\text {oc }}$ is given by the concentration gradient of the same ionic species $\mathrm{C} 1$ on the positive contact and $\mathrm{C} 2$ on the negative contact by the Nernst equation: $V_{o c}=\frac{k T}{q} \ln \left(\frac{c 1}{c 2}\right)$, where $\frac{k T}{q}$ is the thermal voltage. The voltage reaches the highest value when the concentration gradient is maximum. The $\mathrm{V}_{\text {oc }}$ also depends on the water content of the paper and paper material that determines the diffusion rate of the ionic species introduced. The Zika virus becomes entangled and immobilized in the pores of the paper. This results in a space charge region similar to a semiconductor $\mathrm{P}-\mathrm{N}$ junction giving rise to $\mathrm{V}_{\text {oc. }}$

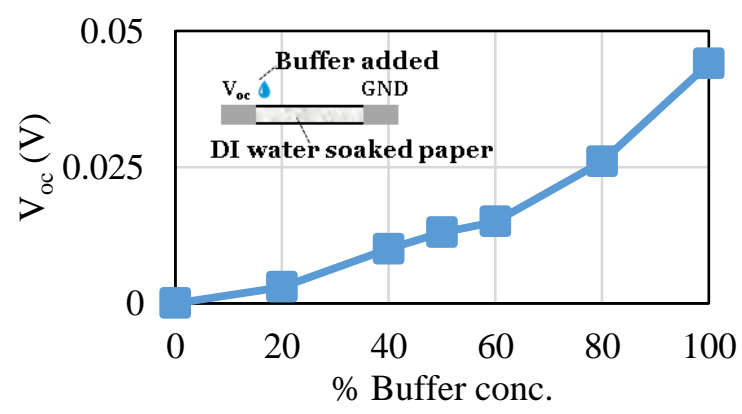

Figure 4. Voc vs percentage volume concentration of buffer with one side of the paper dipped in DI water and the other side with the diluted buffer solution.

The Zika virus has a small residual negative charge (shown in Fig. 5(a)) that reduces the $V_{\text {oc }}$ as soon as it was added to the paper strip. To demonstrate that the Zika (40 nm diameter) was immobilized in the paper, Zika solution was added to a paper device. The buffer diffuses in the paper easily and its concentration gradient diminishes as a function of time. But Zika being immobilized cannot diffuse and the Zika concentration gradient is maintained as shown by the change in $\mathrm{V}_{\text {oc }}$ (Fig. 5). If Zika was also mobile, the resulting $\mathrm{V}_{\text {oc }}$ will diminish as a function of time as the Zika diffuses.

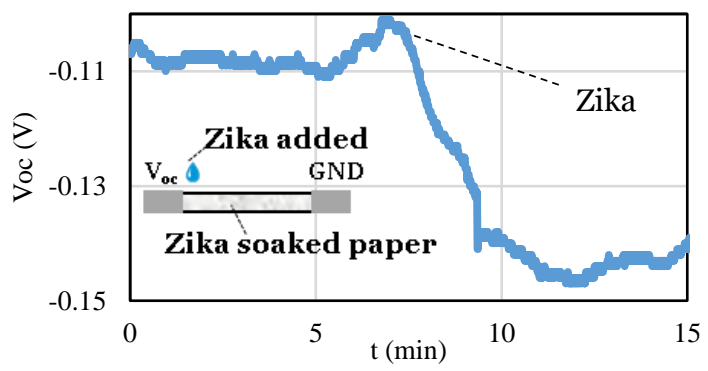

(a)

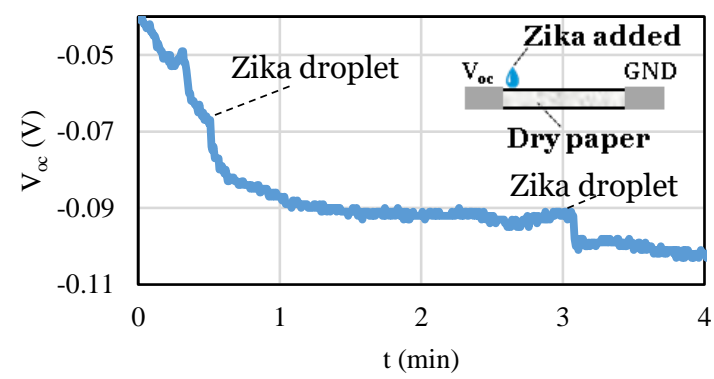

(b)

Figure 5. Zika introduced a) at $\mathrm{t}=7.45 \mathrm{~min}$ to paper device presoaked in Zika to demonstrate net negative charge of the virus and $b$ ) at $t=0.5 \mathrm{~min}$ and $t=3.1 \mathrm{~min}$ to a dry paper device to demonstrate that as the Zika is applied on the positive side, it stays immobilized in the applied region as the buffer spreads maintaining the voltage level. 
Fig. 6 shows the $V_{\text {oc }}$ change $\left(\Delta V_{\text {oc }}\right)$ after adding Zika on the positive side of the aptamer-coated paper device as a function of Zika concentration. Zika-aptamer binding redistributes their charges. Once all the resident aptamers bind with Zika, the only changes in the device charge content and its gradient are introduced by the additional Zika alone that is (slightly) negatively charged. Thus, successive additions of Zika initially introduced larger changes in the device $\mathrm{V}_{\text {oc }}$ followed by smaller changes once all the resident aptamers are saturated as shown in summary in Fig. 6. The average "point" sensitivity was measured to be $2.6 \mathrm{nV} /$ Zika from the average $\Delta \mathrm{V}_{\text {oc }}$ of $\sim 18 \mathrm{mV}$ for $2 \mu \mathrm{l}$ Zika. The sensitivity was nearly the same for $4 \mu \mathrm{l}$ Zika in device P1 but not in device P2 (Fig. 6). The paperbased sensor's output response had a noise voltage of $2 \mathrm{mV}$ and the minimum detectable voltage was $1 \mathrm{mV}$ that resulted in the MDS of $1.2 \times 10^{6} \mathrm{Zika}$.

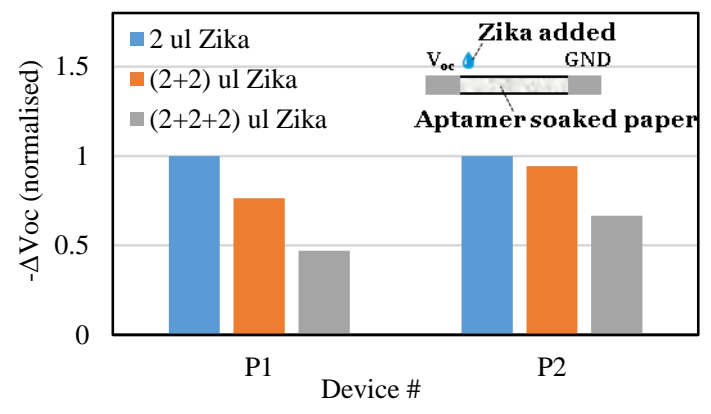

Figure 6. $2 \mu \mathrm{l}$ of diluted Zika added to aptamer-soaked paper at each instance (total 3 times) repeated on 2 separate paper devices (P1, P2).

To verify the presence of aptamers and Zika in the paper-based devices we carried out two additional experiments. The first series of experiments used atomic force microscopy (AFM) to image Zika-coated regions of the paper-based device as shown in Fig. 7(a). Although one can see large Zika complexes, individual Zika viruses could not be resolved. The paper has very large surface roughness in excess of $2 \mu \mathrm{m}$ rms that prevented performing high spatial resolution scans. We then performed AFM scans on aptamers and Zika deposited on gold-coated glass samples. As can be seen in Fig. 7(b) one could resolve individual Zika viruses on gold.

Next, AFM studies using AFM probes with aptamer-functionalized beads were carried on paper-based devices coated with different analytes. These studies showed that the aptamerfunctionalized AFM probe measured larger stiction forces with papers coated with aptamers and then Zika papers with only aptamer or Zika alone. The results are shown in Fig. 7 (d). The stiction force of Zika/aptamer/paper was higher due to aptamer-specific binding with Zika that was anchored to its aptamer anchored to the paper.

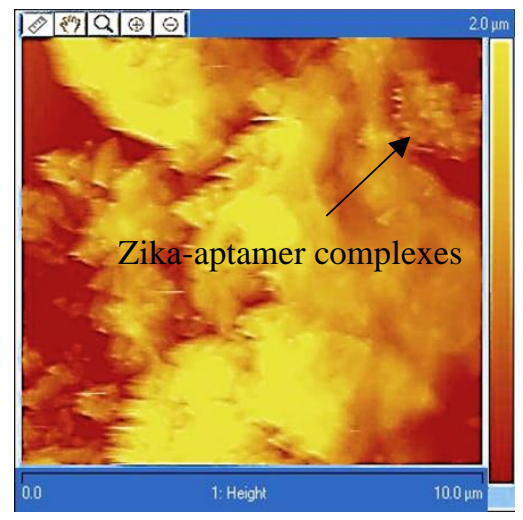

(a)

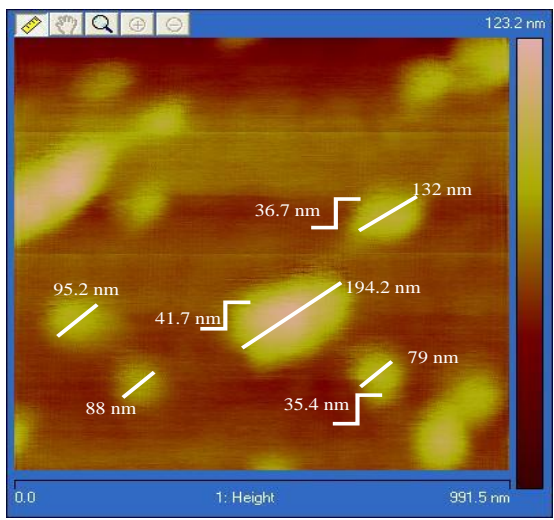

(b) 


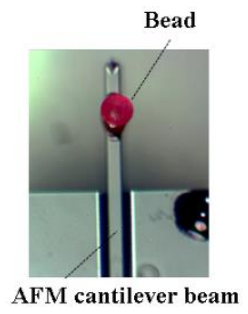

(c)

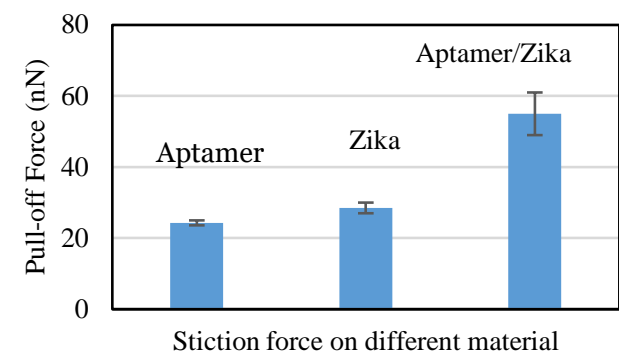

(d)

Figure 7. a) AFM image of the Zika on aptamer-coated paper. The scale is $10 \mu \mathrm{m}$. b) AFM image of Zika on gold substrate showing the Zika step height of $\sim 40 \mathrm{~nm}$ to demonstrate Zika on the aptamercoated gold surface. c) Photograph of the AFM tip with a single microbead (Diameter $150 \mu \mathrm{m}$ ). d) AFM stiction force measured using the AFM tip (c) with bead coated with aptamer specific to Zika. e) Raman spectra of buffer/paper, aptamer/paper, Zika/paper and Zika/aptamer/paper. The spectra are normalized with dry paper Raman spectrum. These spectra clearly show the difference between aptamers, Zika and buffer deposited on paper.

The second set of experiments used Raman spectroscopies on paper-based devices coated with different analytes. The Raman spectra shown in Fig. 8 are from different regions of the paper coated with aptamers, Zika and Zika/aptamers. A DeltaNu spectrometer with Examiner 785 Raman unit was used in these experiments. The amount of aptamers used in coating these samples were $10 \mu \mathrm{l}$ with 1 $\mu \mathrm{M}$ concentrations and the Zika concentration was $1.7 \times 10^{8} / \mu \mathrm{l}$ and the amount used in each application was $1 \mu \mathrm{l}$. The buffer solution was $1 \mu \mathrm{l}$ of $1 \times$ PBS with $1 \mathrm{mM} \mathrm{MgCl}_{2}$. The Raman peak due to Zika was around $1590 \mathrm{~cm}^{-1}$ [34] and [35].

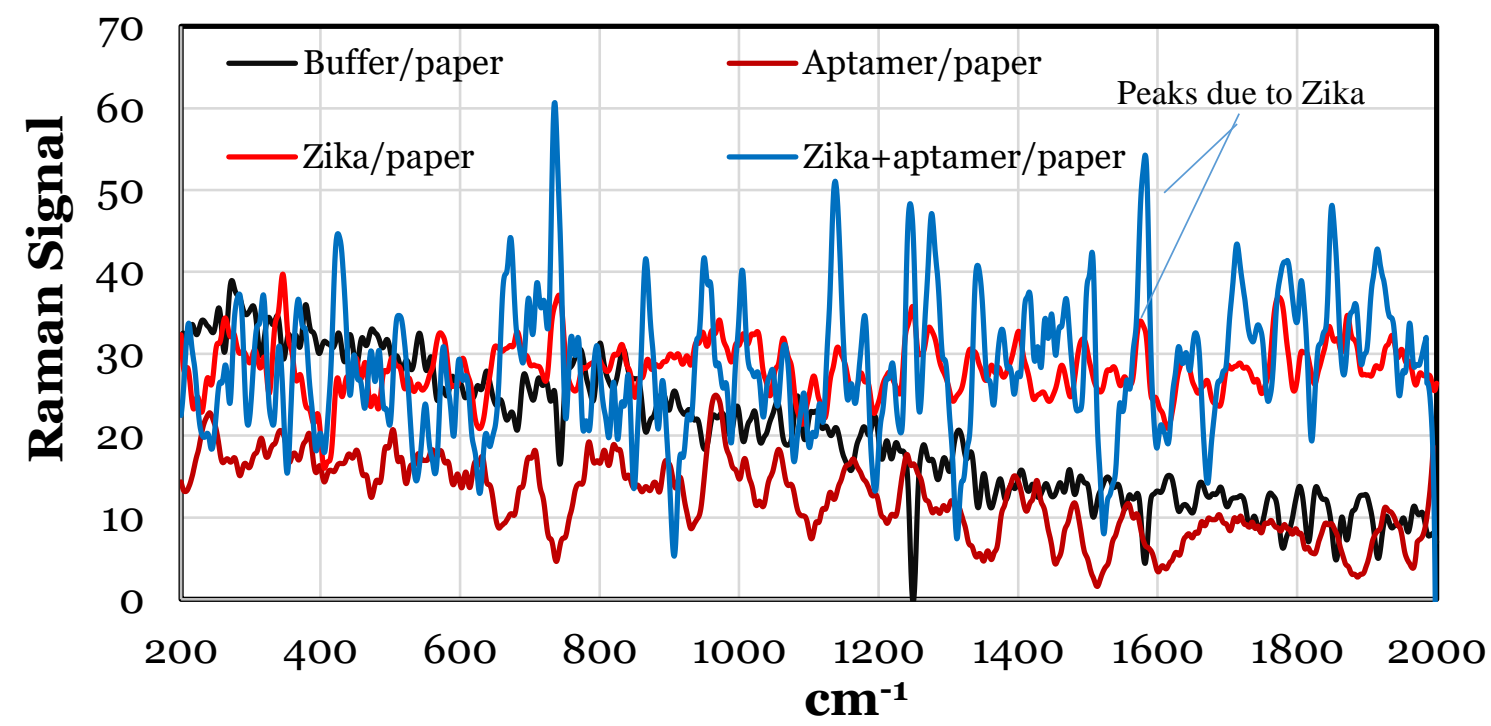

(e)

Figure 8. Raman spectra of buffer/paper, aptamer/paper, Zika/paper and Zika/aptamer/paper. The spectra are normalized with dry paper Raman spectrum. These spectra clearly show the difference between aptamers, Zika and buffer deposited on paper.

We then used a laser printer to print patterns on paper followed by adding silver paint contacts as shown in Fig. 2(a) and Fig. 9 insets. The purpose of the printed device is to clearly show the location of the Zika solution (or fluids that may contain Zika) on the device. Fig. 9(a) shows the Voc-time response for paper-based devices (D1, D2, and D3). The negative $\Delta \mathrm{V}_{\text {oc }}$ shift in these devices, after 
introducing Zika, are shown in Fig. 9(b). We verified that the aptamer stayed and was resident in the paper even after rinsing the paper with DI water. Fig. 9(c) shows the response of adding Zika on the three different aptamer-coated printed devices (D4, D5, D6). The Voc levels in Fig. 9(c) are similar and consistent with that of aptamer-coated device in Fig. 9(b).

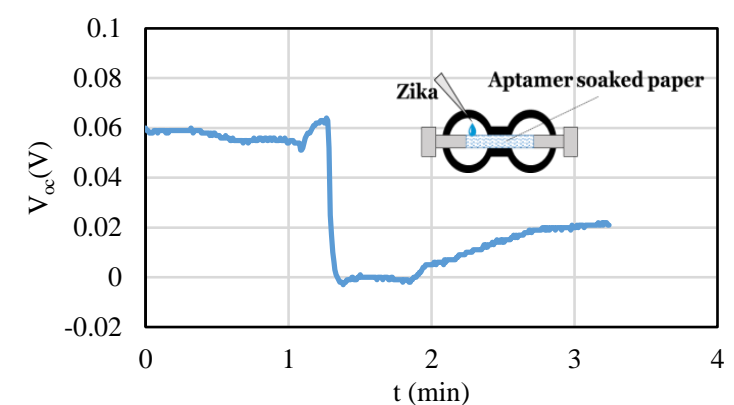

(a)

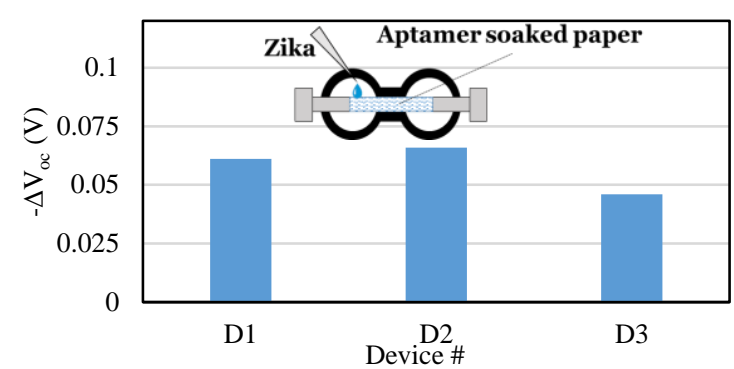

(b)

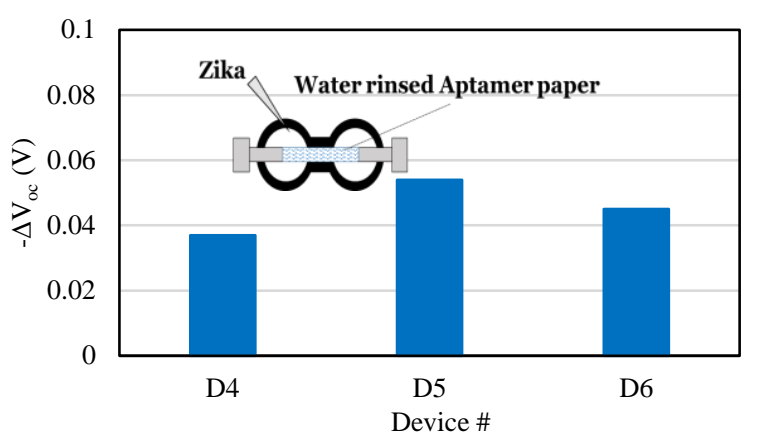

(c)

Figure 9. a) $V_{\text {oc }}$ vs time of the printed sensor with $\mathrm{Zika}$ added at $\mathrm{t}=1.3 \mathrm{~min}$. $\Delta \mathrm{V}_{\text {oc }}$ obtained on adding Zika to b) aptamer coated printed device D1, D2, D3, and c) aptamer coated and subsequently rinsed devices D4, D5 and D6, showing that the aptamer remain immobilized in the paper after rinsing.

The actual interactions between different components in the paper matrix is quite involved and the sensor response depends on the history of the paper, temperature, air current on the sensor, and

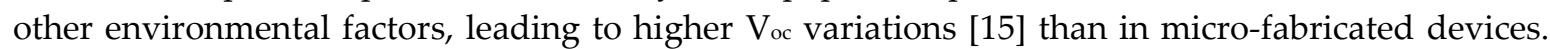
However, the results shown here are in agreement with the overall trends seen with many averaged measurement results (with a standard deviation of $\sim 2 \mathrm{mV}$ ) and theory. Also, the paper-based devices were manually cut $(\sim 1 \mathrm{~cm} \times 0.2-0.3 \mathrm{~cm})$ with uncertain dimensions. Variations in device dimensions can be considerably reduced using automated machine cutting. Uniformity of the electrical contacts can also be a concern that can be addressed using 3-D printing. Another very important source of error was in making sure that the paper sensor was not saturated with the analytes introduced at each step. When the paper was saturated, the addition of Zika resulted in a thin layer of liquid above the paper sensor and Zika did not incorporate in the paper resulting in noisy sensor output. The sensor was also very sensitive to spurious environmental electric fields generated by us and other objects nearby that could be addressed by proper grounding.

\section{Electronic Readout}

We next explored the possibility of detecting the virus from the paper-based sensor in a form of direct electronic readout through an LCD instead of measuring the open circuit voltage using a voltmeter. The LCD can be configured to display a letter such as " $Z$ " once the paper sensor voltage reaches a threshold value generated by the presence of sufficient number of Zika viruses. In order to demonstrate the feasibility of such application, we used an LCD screen from a digital watch and experimentally obtained turn-on voltage for a single display segment to be $\sim 100 \mathrm{mV}$. From the previous results we observed that addition of Zika resulted in $\Delta \mathrm{V}_{\text {oc }} \sim 50 \mathrm{mV}$. Thus, 6 paper sensors 
were connected in series to generate $\sim 300 \mathrm{mV}$ Fig. 10(a) that is sufficient to power may readout devices including the LCD. The only drawbacks are that the series-connected sensors should be fed Zika separately and the external load should not draw more than $0.1 \mathrm{~mA}$ current to cause internal sensor resistor to become important. As can be seen in Fig. 10(b), once the Zika was added, the LCD displayed "A". The LCD can be replaced by electronic-ink display which requires much lower voltages/power eliminating the necessity of using many paper-based devices in series.

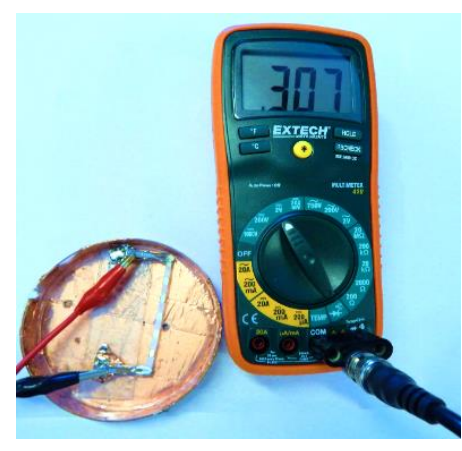

(a)

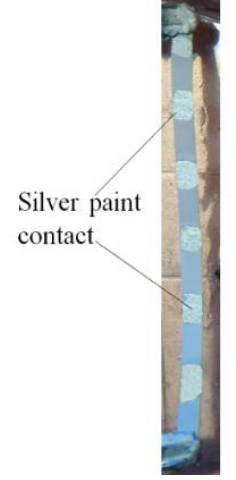

Before adding Zika Virus

(b)

Figure 10. a) Output from the paper sensor measured using multimeter showing voltage level of $\sim 300 \mathrm{mV}$ for 6 paper sensors connected in series. b) Output from the paper sensor connected to the LCD readout optioned from digital watch showing the letter " $\mathrm{A}$ " on adding Zika on each side of each of the aptamer-soaked paper devices. The paper strip is connected using clips with 6 devices imprinted on the strip. The grey patches are the silver contact paint regions separating the sensors from each other.

The paper-based sensors we constructed and reported here were very reproducible as long as they were not saturated and as long as they were grounded properly. Their "point" sensitivity of 2.6 $\mathrm{nV} / \mathrm{Zika}$ and their minimum detectable signal (MDS) of $1.2 \times 10^{6} \mathrm{Zika}$ are quite good given their simple structures and ease of operations. Selectivity of this sensor as well as other aptamer-based sensors are limited by the selectivity of their respective aptamers that can be modified to achieve better selectivity if needed. In the case of paper-based sensors discussed here, the selectivity may also be affected by viruses becoming immobilized in the paper even if they are do not bind with the resident aptamer. These other viruses can be removed by mildly washing them away that will not affect the Zika-aptamer complexes appreciably. The real-world application of Zika paper sensors will involve presence of bodily fluids such as urine or sweat or saliva. These biofluids are very complex and proper filtering on the sensor will be used to remove their components that may affect the paper sensor operation adversely.

As with any sensor development, the research and development efforts have many different stages. It usually starts with developing the transduction mechanism using modified "clean" samples and progresses toward more realistic samples in real world. The present work describes the transduction phenomena of "sensing pure Zika" virus using a paper-based potentiometric device that to the best of our knowledge was not done before. The innovation has two parts: a) using paper-based sensors to detect the whole virus and b) using resident aptamers in the paper to bind with Zika. The sensor's selectivity comes from its resident aptamer. In the near future we will measure and report our sensors' selectivity and verify their operations in the presence of bodily fluid.

\section{Conclusion}

This work demonstrates for the first time a paper-based sensor for detecting Zika virus using potentiometry. The sensor had a minimum sensitivity of $2.6 \mathrm{nV} / \mathrm{Zika}$ and a minimum detectable 
signal of $1.2 \times 10^{6} \mathrm{Zika}$. It can be generalized to detect any other viruses, pathogens even bacteria by simply using appropriate aptamers. The most important and unique aspect of the potentiometric paper based Zika virus is its structural simplicity and ease of use. This study demonstrates the feasibility of detecting whole Zika viruses and the use of simple electronic readout using paper-based sensors. Although aptamers are known for their high specificity to capture target pathogens and viruses, more study is required with different viruses to verify and measure its cross-sensitivity.

Acknowledgments: This research was supported by a National Science Foundation Grant under Dr. Leon Esterowitz.

\section{References}

[1] A. Määttänen et al., "A low-cost paper-based inkjet-printed platform for electrochemical analyses," Sensors Actuators, B Chem., vol. 177, pp. 153-162, 2013.

[2] A. C. Siegel, S. T. Phillips, M. D. Dickey, N. Lu, Z. Suo, and G. M. Whitesides, "Foldable printed circuit boards on paper substrates," Adv. Funct. Mater., vol. 20, no. 1, pp. 28-35, Jan. 2010.

[3] D. Tobjörk and R. Österbacka, "Paper electronics," Adv. Mater., vol. 23, no. 17, pp. 19351961, May 2011.

[4] M. Novell, T. Guinovart, P. Blondeau, F. X. Rius, and F. J. Andrade, "A paper-based potentiometric cell for decentralized monitoring of Li levels in whole blood," Lab Chip, vol. 14, no. 7, pp. 1308-1314, Mar. 2014.

[5] J. Cui, G. Lisak, S. Strzalkowska, and J. Bobacka, "Potentiometric sensing utilizing paperbased microfluidic sampling," Analyst, vol. 139, no. 9, pp. 2133-2136, Apr. 2014.

[6] J. Szucs and R. E. Gyurcsányi, "Towards Protein Assays on Paper Platforms with Potentiometric Detection," Electroanalysis, vol. 24, no. 1, pp. 146-152, Jan. 2012.

[7] J. Hu, A. Stein, and P. Bühlmann, “A Disposable Planar Paper-Based Potentiometric IonSensing Platform," Angew. Chemie - Int. Ed., vol. 55, no. 26, pp. 7544-7547, Jun. 2016.

[8] P. Sjöberg et al., "Paper-based potentiometric ion sensors constructed on ink-jet printed gold electrodes," Sensors Actuators, B Chem., vol. 224, pp. 325-332, 2016.

[9] E. W. Nery and L. T. Kubota, "Integrated, paper-based potentiometric electronic tongue for the analysis of beer and wine," Anal. Chim. Acta, vol. 918, pp. 60-68, Apr. 2016.

[10] J. H. Jin, J. H. Kim, S. K. Lee, S. J. Choi, C. W. Park, and N. K. Min, “A fully integrated papermicrofluidic electrochemical device for simultaneous analysis of physiologic blood ions," Sensors (Switzerland), vol. 18, no. 1, 2018.

[11] J. Kofler, S. Nau, and E. J. W. List-Kratochvil, "A paper based, all organic, referenceelectrode-free ion sensing platform," J. Mater. Chem. B, vol. 3, no. 25, pp. 5095-5102, 2015.

[12] J. Ding, N. He, G. Lisak, W. Qin, and J. Bobacka, "Paper-based microfluidic sampling and separation of analytes for potentiometric ion sensing," Sensors Actuators, B Chem., vol. 243, pp. 346-352, 2017.

[13] M. Parrilla, R. Cánovas, and F. J. Andrade, "Paper-based enzymatic electrode with enhanced potentiometric response for monitoring glucose in biological fluids," Biosens. Bioelectron., vol. 90, pp. 110-116, 2017.

[14] G. Lisak, J. Cui, and J. Bobacka, “Paper-based microfluidic sampling for potentiometric 
determination of ions," Sensors Actuators, B Chem., vol. 207, no. PB, pp. 933-939, 2015.

[15] R. Kawahara, P. Sahatiya, S. Badhulika, and S. Uno, "Paper-based potentiometric pH sensor using carbon electrode drawn by pencil," Jpn. J. Appl. Phys., vol. 57, no. 4, p. 04FM08, Apr. 2018.

[16] J. Ping, Y. Wang, K. Fan, W. Tang, J. Wu, and Y. Ying, "High-performance flexible potentiometric sensing devices using free-standing graphene paper," J. Mater. Chem. B, vol. 1, no. 37, pp. 4781-4791, 2013.

[17] A. A. Al-Qahtani, N. Nazir, M. R. Al-Anazi, S. Rubino, and M. N. Al-Ahdal, “Zika virus: a new pandemic threat," J Infect Dev Ctries, vol. 10, no. 3, pp. 201-207, 2016.

[18] C. J. Haug, M. P. Kieny, and B. Murgue, "The zika challenge," New England Journal of Medicine, vol. 374, no. 19. Massachussetts Medical Society, pp. 1801-1803, 12-May-2016.

[19] J. Shin, A. G. Cherstvy, and R. Metzler, "Sensing Viruses by Mechanical Tension of DNA in Responsive Hydrogels," APS, vol. 4, no. 2, 2014.

[20] K. Murray, R. Gorchakov, ... A. C.-E. infectious, and undefined 2017, "Prolonged detection of Zika virus in vaginal secretions and whole blood," ncbi.nlm.nih.gov.

[21] G. Calvet, R. Aguiar, A. Melo, ... S. S.-T. L. infectious, and undefined 2016, “Detection and sequencing of Zika virus from amniotic fluid of fetuses with microcephaly in Brazil: a case study," Elsevier.

[22] O. Faye, O. Faye, A. Dupressoir, ... M. W.-J. of C., and undefined 2008, “One-step RT-PCR for detection of Zika virus," Elsevier.

[23] "Detection of Zika virus in urine," ncbi.nlm.nih.gov.

[24] C. Klungthong et al., "Dengue Virus Detection Using Whole Blood for Reverse Transcriptase PCR and Virus Isolation," J. Clin. Microbiol., vol. 45, no. 8, pp. 2480-2485, 2007.

[25] "Detection of Zika virus in saliva," Elsevier.

[26] K. H. Lee and H. Zeng, “Aptamer-Based ELISA Assay for Highly Specific and Sensitive Detection of Zika NS1 Protein," Anal. Chem., vol. 89, no. 23, pp. 12743-12748, 2017.

[27] R. D. Jenison, S. C. Gill, A. Pardi, and B. Polisky, "High-resolution molecular discrimination by RNA," Science (80-. )., vol. 263, no. 5152, pp. 1425-1429, 1994.

[28] E. Bakker, P. Bühlmann, and E. Pretsch, "The phase-boundary potential model," Talanta, vol. 63, no. 1, pp. 3-20, 2004.

[29] H. H.-C. E. Science and undefined 1970, "The liquid junction potential-the free diffusion junction," Elsevier.

[30] H. W. Harper, "Calculation of liquid junction potentials," J. Phys. Chem., vol. 89, no. 9, pp. 1659-1664, 1985.

[31] “Zika Envelope Recombinant Antigen I Zika Virus | ProSpec." [Online]. Available: https://www.prospecbio.com/zika_envelope_sf9.

[32] S. Dolai and M. Tabib-Azar, “433 MHz Lithium Niobate microbalance aptamer-coated whole Zika virus sensor with $370 \mathrm{~Hz} / \mathrm{ng}$ sensitivity," IEEE Sens. J., pp. 1-1, Dec. 2019.

[33] "Base Pair Biotechnologies - Aptamer Discovery Company." [Online]. Available: https://www.basepairbio.com/. [Accessed: 04-Sep-2019].

[34] M. Sanchez-Purra, M. Carre-Camps, H. de Puig, I. Bosch, L. Gehrke, and K. Hamad-Schifferli, "Surface-Enhanced Rama Spectroscopy-Based Sandwich Immunoassays for Multiplexed Detection of Zika and Dengue Viral Biomarkers." ACS Infectious Diseases, 2017, 3, 767-776. DOI:

10.1021/acsinfecdis.7b00110. 